\title{
Combining baseline TMTV and gene profiling for a better risk stratification in diffuse large B cell lymphoma
}

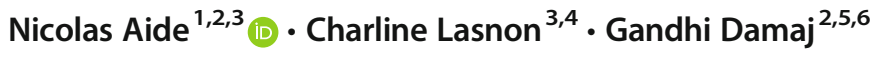 \\ Received: 22 January 2018 / Accepted: 25 January 2018 / Published online: 17 February 2018 \\ (C) Springer-Verlag GmbH Germany, part of Springer Nature 2018
}

Diffuse large B cell lymphoma (DLBCL) is the most common type of mature B-cell non-Hodgkin lymphoma, accounting for approximately one-third of all cases. This is a heterogeneous disease with different prognostic patterns. Patients benefit from immuno-chemotherapy; however, 30 to $40 \%$ of patients are refractory to it or rapidly relapse after first-line treatment, emphasizing the need to better characterize these patients.

Many prognostic scores have been elaborated over time to better categorize patients into different risk groups. Although the International Prognostic Index (IPI) is the most used scoring system at diagnosis in clinical practice [1], it fails to characterize the biological and molecular patterns of the disease and thus patients' outcomes [2].

Immunohistochemical (IHC) algorithms have been used for risk stratification, according to the cell of origin (COO). It categorizes patients into germinal center B-cell like (GCB)DLBCL, activated B-cell like (ABC)-DLBCL, about 10-15\% of cases being unclassified DLBCL subtypes. Patients with the GCB subtype usually have better prognosis than patients with the $\mathrm{ABC}$ subtype. Although $\mathrm{COO}$ is useful for predicting patients' outcome, the GCB and $\mathrm{ABC}$ subtypes remain heterogeneous, with better and worse prognostic subsets within

This Editorial Commentary refers to the article https://oi.org/10.1007/ s00259-017-3907-x.

Nicolas Aide

aide-n@chu-caen.fr

1 Nuclear Medicine Department, Caen University Hospital, Caen, France

2 Normandie University, Caen, France

3 INSERM 1086 ANTICIPE, Normandie University, Caen, France

4 Nuclear Medicine Department, François Baclesse Cancer Centre, Caen, France

5 Haematology Institute, Caen University Hospital, Caen, France

6 MICAH INSERM U1245, Rouen University, Rouen, France each group. On the other hand, clinical studies investigating the capacity of $\mathrm{COO}$ by IHC algorithms in determining the efficacy of certain therapies showed conflicting results, which is partly due to the IHC techniques used.

A major advance in the determination of $\mathrm{COO}$ was the application of gene expression profiling (GEP), which facilitates the identification of multiple genetic abnormalities in DLBCL.

GEP can be performed using several molecular biology techniques. GEP used in formalin-fixed paraffin-embedded tissue (Affymetrix) [3] is probably the preferred method, and the digital gene expression-based assay using NanoString technology (Lymph2Cx) [4] shows promising results. However, these techniques are not yet available in most centres, expensive, and not used in routine practice. The reverse transcriptase-multiplex ligation-dependent probe-amplification (RT-MLPA) on frozen sections of the tumour specimen showed a good correlation with the Affymetrix technique, the gold standard classification in determining the $\mathrm{COO}$ of DLBCL [5]. RT-MLPA is cost effective and rapid to implement in routine practice.

FDG PET is now routinely used for baseline staging and response assessment in DLBCL [6]. Retrospective studies have shown that a high total metabolic tumour volume (TMTV) on baseline PET could be used as a prognosticator [7]. There is however to date no consensus on the method to be used to draw TMTV, and the different contouring methods available have been shown to affect TMTV and therefore to provide different thresholds for patients' risk stratification. It should be noted that Cottereau et al. [8] showed in another NHL subtype that despite providing different thresholds, TMTV remains a strong prognosticator whatever the contouring method used. However, their study was performed using EARL-compliant data in four out of five centres, and the same TMTV delineation for the whole series of patients. Applying different contouring method for each centre in the multicentre setting would probably affect the capability of FDG PET to predict patients' outcome. TMTV is also strongly 
affected by the newer reconstruction algorithms $[9,10]$. These issues are exemplified by the figure below. Figure 1.

In this issue of the EJNMMI, Toledano et al. [11] present a retrospective analysis of baseline TMTV in 114 DLBCL patients. TMTV was computed using the $41 \%$ isocontour method recommended by the EANM guidelines and combined to GEP using RT-MLPA to predict patients' outcome. Their study showed that the combination of TMTV and GEP led to the identification of three subgroups harbouring strikingly different prognoses: (i) patients with low $\left(<261 \mathrm{~cm}^{3}\right)$ TMTV, whatever the GEP, (ii) high TMTV $\left(>261 \mathrm{~cm}^{3}\right)$ and BCB phenotype, and (iii) high TMTV and ABC phenotype. The 5 -year progression-free survival (PFS) of these patients was $72 \%, 51 \%$, and $17 \%$ respectively, and both TMTV and GEP were found to be independent prognosis factors for PFS.

One can question how and when this combination of two biomarkers so far unused in clinical routine will make its way into our daily practice to achieve our goal of a tailored medicine: the identification of patients with the worst prognosis who could benefit from alternative therapeutic strategies. Confirmation of Toledano and colleagues' findings will require larger series of patients, pooling data from different centres. Because of the issues mentioned above, this confirmation will need harmonization of PET methodology, from acquisition/reconstruction to TMTV contouring using an automatic or semi-automatic (to minimize inter- and intraobserver variability as much as possible), reliable, and userfriendly contouring tool. The last item is not trivial; computation of TMTV will certainly be accepted by the PET community in routine practice only if it minimally affects the time required for PET reporting in busy PET centres. While we know for sure that standardization of the methodology used to delineate TMTV is warranted, one can wonder how much standardization is needed for GEP and which technique is
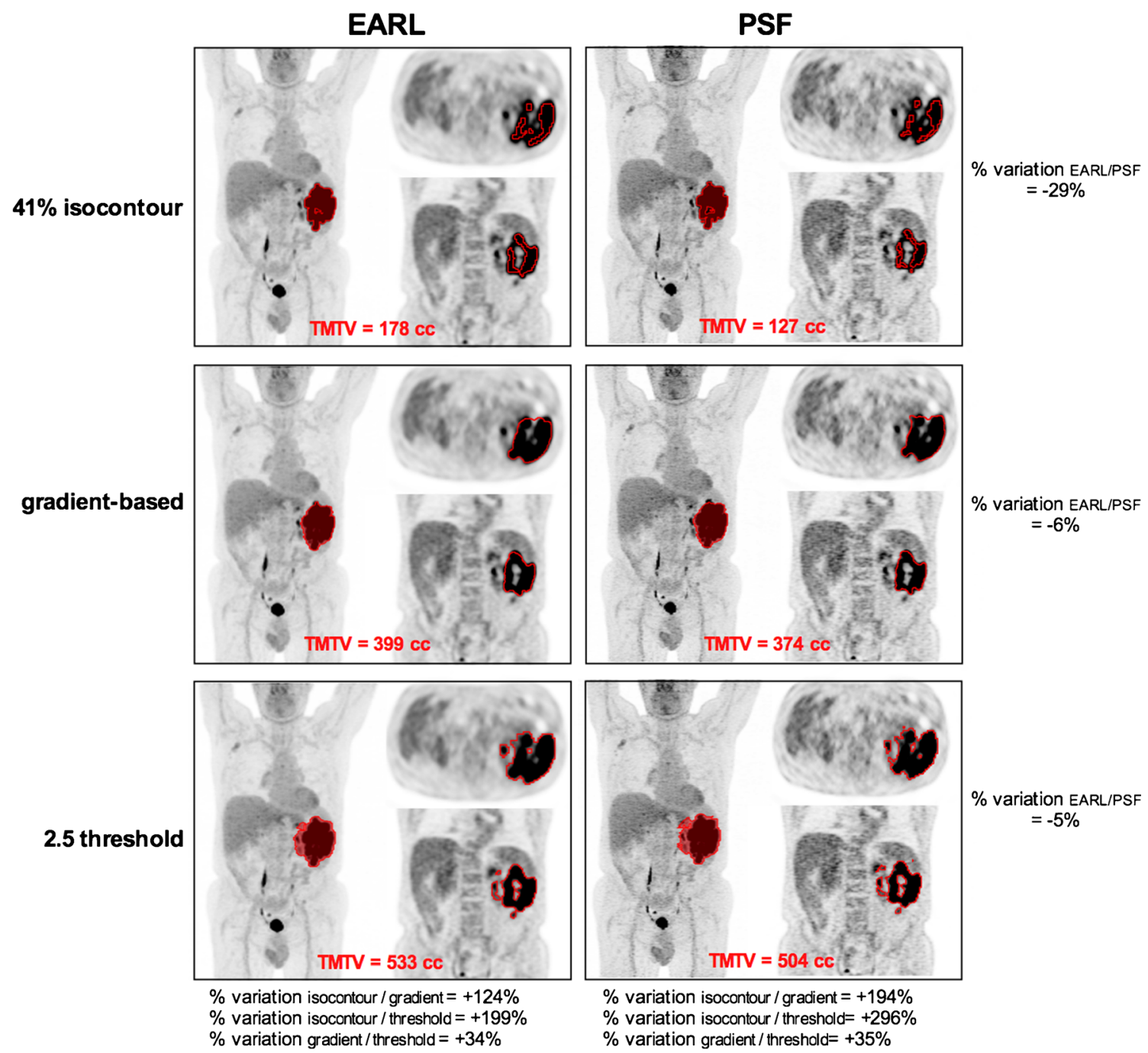

$\%$ variation isocontour $/$ gradient $=+194 \%$

$\%$ variation isocontour $/$ threshold $=+296 \%$

$\%$ variation gradient $/$ threshold $=+35 \%$

Fig. 1 Representative examples of TMTV delineation using the $41 \%$ isocontour method recommended by the EANM, a $\mathrm{SUV}_{\max }=2.5$ threshold or a more advanced gradient based method in EARL

compliant images (left panels) or images reconstructed with unfiltered point spread function (PSF) reconstruction (right panels) 
likely to be used on a large scale. RT-MLPA, the GEP technique used by Toledano et al. to determine the COO of DLBCL, is cost-effective and rapid to implement in routine practice.

Finally, evaluation of the combination of several biomarkers to predict PFS or OS using the Cox model implies statistical considerations, given that the number of patients required to reach an adequate statistical power depends on the number of events (relapse for PFS and death for OS) and the number of variables (biomarkers and other known prognostic factors such as the IPI score). Hence, larger series of patients would open the way to multivariable analysis with an adequate statistical power or even the use of machine-learning methodology [12], using multiple PET metrics such as total lesion glycolysis (TLG), and tumour heterogeneity using textural features (TFs) [13]. These features are extracted from the TMTV and are also known to be sensitive to reconstruction parameters [14], highlighting, again, the need for a standardization of PET data.

In conclusion, the study from Toledano et al. paves the way for the combination of FDG PET metrics with a simple molecular technique to determine the prognosis and eventually tailor the therapy of patients with DLBCL. To this end, a consensual TMTV delineation procedure is mandatory.

\section{Compliance with ethical standards}

Conflicts of interest None to declare.

Ethical approval Not applicable.

\section{References}

1. Ziepert M, Hasenclever D, Kuhnt E, Glass B, Schmitz N, Pfreundschuh M, et al. Standard international prognostic index remains a valid predictor of outcome for patients with aggressive CD20+ B-cell lymphoma in the rituximab era. J Clin Oncol. 2010;28:2373-80. https://doi.org/10.1200/jco.2009.26.2493.

2. Choi WW, Weisenburger DD, Greiner TC, Piris MA, Banham AH, Delabie J, et al. A new immunostain algorithm classifies diffuse large B-cell lymphoma into molecular subtypes with high accuracy. Clin Cancer Res. 2009;15:5494-502. https://doi.org/10.1158/10780432.ccr-09-0113.

3. Wright G, Tan B, Rosenwald A, Hurt EH, Wiestner A, Staudt LM. A gene expression-based method to diagnose clinically distinct subgroups of diffuse large B cell lymphoma. Proc Natl Acad Sci
U S A. 2003;100:9991-6. https://doi.org/10.1073/pnas. 1732008100 .

4. Scott DW, Wright GW, Williams PM, Lih CJ, Walsh W, Jaffe ES, et al. Determining cell-of-origin subtypes of diffuse large B-cell lymphoma using gene expression in formalin-fixed paraffin-embedded tissue. Blood. 2014;123:1214-7. https://doi.org/10.1182/ blood-2013-11-536433.

5. Mareschal S, Ruminy P, Bagacean C, Marchand V, Cornic M, Jais JP, et al. Accurate classification of germinal Center B-cell-like/activated B-cell-like diffuse large B-cell lymphoma using a simple and rapid reverse transcriptase-multiplex ligation-dependent probe amplification assay: a CALYM study. J Mo Diagn. 2015; https:// doi.org/10.1016/j.jmoldx.2015.01.007.

6. Nanni C, Cottereau AS, Lopci E, Bodet-Milin C, Coronado M, Pro $\mathrm{B}$, et al. Report of the 6th international workshop on PET in lymphoma. Leuk Lymphoma. 2017;58:2298-303. https://doi.org/10. 1080/10428194.2017.1298752.

7. Sasanelli M, Meignan M, Haioun C, Berriolo-Riedinger A, Casasnovas RO, Biggi A, et al. Pretherapy metabolic tumour volume is an independent predictor of outcome in patients with diffuse large B-cell lymphoma. Eur J Nucl Med Mol Imaging. 2014;41: 2017-22. https://doi.org/10.1007/s00259-014-2822-7.

8. Cottereau AS, Hapdey S, Chartier L, Modzelewski R, Casasnovas $\mathrm{O}$, Itti E, et al. Baseline total metabolic tumor volume measured with fixed or different adaptive thresholding methods equally predicts outcome in peripheral T cell lymphoma. J Nucl Med. 2017;58: 276-81. https://doi.org/10.2967/jnumed.116.180406.

9. Aide N, Lasnon C, Veit-Haibach P, Sera T, Sattler B, Boellaard R. EANM/EARL harmonization strategies in PET quantification: from daily practice to multicentre oncological studies. Eur J Nucl Med Mol Imaging. 2017;44:17-31. https://doi.org/10.1007/ s00259-017-3740-2.

10. Lasnon C, Enilorac B, Popotte H, Aide N. Impact of the EARL harmonization program on automatic delineation of metabolic active tumour volumes (MATVs). EJNMMI Res. 2017;7:30. https:// doi.org/10.1186/s13550-017-0279-y.

11. Toledano MN, Desbordes P, Banjar A, Gardin I, Vera P, Ruminy P, et al. Combination of baseline FDG PET/CT total metabolic tumour volume and gene expression profile have a robust predictive value in patients with diffuse large B-cell lymphoma. Eur J Nucl Med Mol Imaging. 2018; https://doi.org/10.1007/s00259-017-3907-x.

12. Oliveira FPM, Faria DB, Costa DC, Castelo-Branco M, Tavares JMRS. Extraction, selection and comparison of features for an effective automated computer-aided diagnosis of Parkinson's disease based on [123I]FP-CIT SPECT images. Eur J Nucl Med Mol Imaging. 2017; https://doi.org/10.1007/s00259-017-3918-7.

13. Aide N, Talbot M, Fruchart C, Damaj G, Lasnon C. Diagnostic and prognostic value of baseline FDG PET/CT skeletal textural features in diffuse large B cell lymphoma. Eur J Nucl Med Mol Imaging. 2017; https://doi.org/10.1007/s00259-017-3899-6.

14. Lasnon C, Majdoub M, Lavigne B, Do P, Madelaine J, Visvikis D, et al. (18)F-FDG PET/CT heterogeneity quantification through textural features in the era of harmonisation programs: a focus on lung cancer. Eur J Nucl Med Mol Imaging. 2016;43:2324-35. https:// doi.org/10.1007/s00259-016-3441-2. 